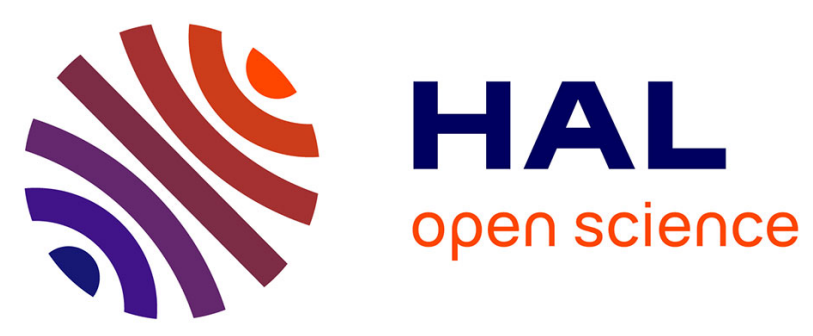

\title{
Uplink Coverage and Handoff Rate with Realistic Power Control Models and Blind Cell Search
}

\author{
Yassine Hmamouche, Mustapha Benjillali, Samir Saoudi, Halim \\ Yanikomeroglu
}

\section{- To cite this version:}

Yassine Hmamouche, Mustapha Benjillali, Samir Saoudi, Halim Yanikomeroglu. Uplink Coverage and Handoff Rate with Realistic Power Control Models and Blind Cell Search. PIMRC 2020: IEEE 31st Annual International Symposium on Personal, Indoor and Mobile Radio Communications, Aug 2020, London, United Kingdom. 10.1109/PIMRC48278.2020.9217209 · hal-02876545

\section{HAL Id: hal-02876545}

\section{https://imt-atlantique.hal.science/hal-02876545}

Submitted on 21 Jun 2020

HAL is a multi-disciplinary open access archive for the deposit and dissemination of scientific research documents, whether they are published or not. The documents may come from teaching and research institutions in France or abroad, or from public or private research centers.
L'archive ouverte pluridisciplinaire HAL, est destinée au dépôt et à la diffusion de documents scientifiques de niveau recherche, publiés ou non, émanant des établissements d'enseignement et de recherche français ou étrangers, des laboratoires publics ou privés. 


\title{
Uplink Coverage and Handoff Rate with Realistic Power Control Models and Blind Cell Search
}

\author{
Yassine Hmamouche ${ }^{1,2}$, Mustapha Benjillali ${ }^{2}$, Samir Saoudi ${ }^{1}$, and Halim Yanikomeroglu ${ }^{3}$ \\ ${ }^{1}$ IMT Atlantique, Lab-STICC, UBL, 29238 Brest, France \\ Emails: \{yassine.hmamouche; samir.saoudi\}@imt-atlantique.fr \\ ${ }^{2}$ Communication Systems Department, INPT, Rabat, Morocco \\ Email: benjillali@ieee.org \\ ${ }^{3}$ Department of Systems and Computer Engineering, Carleton University, Ottawa, ON K1S 5B6, Canada. \\ Email: halim@sce.carleton.ca.
}

\begin{abstract}
In this paper, we characterize, based on stochastic geometry, the uplink coverage probability with a unified power control scheme built upon realistic path loss models and user equipment (UE) constrained transmit power. To improve their uplink connectivity, active UEs are next assumed to move in a random direction without prior knowledge of their nearest base station location, namely the blind cell search (BCS) movement. A tractable expression of the uplink handoff rate is then derived and the induced uplink coverage probability following the BCS movement is evaluated. The results show different echoes of the uplink coverage probability depending on the serving UE profile (stationary or mobile) and the considered path loss model, which suggests new insights into the design of uplink system parameters.
\end{abstract}

\section{INTRODUCTION}

With the exponential growth of mobile-broadband data usage, driven essentially by means of enhanced device capabilities and emerging data-hungry applications, operators are in a steady race to provide optimal quality of experience (QoE). One key measure to quantify the users' QoE is the time-to-content (TTC) metric, defined as the period time from requesting to receiving online content on the UE display. Typically, it has been observed that users need generally to feel a TTC below 6 seconds to report a positive QoE feedback [1]. Also, downlink performance is commonly known as the first factor affecting the overall TTC since most popular applications download more data than they upload. However, recent measurements have shown that a reduced uplink speed of fewer than $300 \mathrm{kbps}$, may be systematically the bottleneck of a delayed TTC of more than 4 seconds [1]. Hence, the ever-increasing importance of improving uplink coverage.

One effective way for uplink coverage enhancement is by deploying ultra-dense networks (UDNs), envisioned as the workhorse of ubiquitous coverage in fifth-generation (5G) networks and beyond (B5G) [2]. That is, real deployment of nodes in the context of UDNs is opportunistic due to several socio-economic factors, and analytical tools such as stochastic geometry (SG) and the theory of point processes are more efficient to capture such spatial variability of nodes [3], [4].

This work was funded in part by a research grant from Pôle de Recherche Avancée en Communications (PRACOM), and in part by the Regional Council of Brittany, France.
To the authors' knowledge, the work in [5] is the first to consider a tractable SG-based model to evaluate the uplink coverage probability at the level of a typical base station (BS), uniformly and randomly deployed in the Voronoi cell of the serving UE. In [4], the previous work is slightly extended, where the assumption of modelling the active uplink UEs with a Poisson point process (PPP) having the same density as the PPP of BSs, is particularly validated via simulations. In [6], uplink heterogeneous cellular networks based on fractional power control with maximum transmit power at UEs are investigated. In [7], the UEs transmit power is conceived as a random variable mapped via a truncated channel inversion power control to the distribution of the desired link distance. However, the question of uplink UDNs has not been explicitly addressed in the previous works, where all of them have considered the simplistic standard path loss model that has demonstrated less-realistic performance trends in the context of downlink UDNs [8], [9]. Interestingly, the authors of [10] addressed such limitation and evaluated the uplink coverage in UDNs with stationary UEs and a revisited path loss model related to a piecewise function. However, incorporating UEs mobility in the context of UDNs is so crucial given the reduced size of cells.

A review of SG mobility-aware models, shows that there are particularly two directions of analysis: i) the trajectorybased handoff wherein the handoff event occurs as long as the receiver crosses transmitters cell boundary, and hence, the handoff rate is biased by the efficiency of quantifying the statistical distribution of cells boundaries. A representative uplink analysis using such method is given in [11]. ii) The association-based handoff where the handoff event occurs as long as another BS verifies the association criterion better than the current serving BS [12]. To the authors' knowledge, this paper is the first work that extends the association-based handoff concept to the uplink analysis.

Typically, the contributions of this paper are threefold: i) We extend the frameworks in [4]-[7] by evaluating the uplink coverage probability under a unified power control scheme built upon realistic path loss models and constrained transmit power of UEs. ii) Inspired from the the third generation partnership project (3GPP) simulation mobility model [13], active 
UEs are assumed to intuitively engage in a linear movement with a random direction in order to improve their connectivity conditions. In such a context, we derive the uplink handoff rate as an extension of the tractable downlink analysis in [12]. iii) Using the obtained handoff rate, we also evaluate the induced uplink coverage probability following such mobility model. The analytical accuracy of our results is next validated via simulations and the interplay of system parameters with the uplink coverage probability and the handoff rate is assessed.

\section{SYSTEM MODEL}

\section{A. Cellular Network Model and Association Scheme}

We consider the uplink of a cellular network, wherein the location of BSs and users is modeled with respective 2D homogeneous PPPs $\Psi_{\mathrm{b}}$ and $\Psi_{\mathrm{u}}$, with respective densities $\lambda_{\mathrm{b}}$ and $\lambda_{\mathrm{u}}$. Without loss of generality, and as permitted by the Slivnyak-Mecke's theorem [3, Th. 1.4.5], the typical BS at the origin $O$, is taken as the object of the analysis.

We assume an orthogonal access scheme, e.g., OFDMA, where the typical BS schedules randomly one UE per resource block from the UEs located inside its Voronoi cell. We focus on the loaded regime where each BS is active in the uplink, i.e., $\lambda_{\mathrm{u}} \gg \lambda_{\mathrm{b}}$. That is, the process of active UEs (those scheduled to serve their own BS), denoted by $\Psi \subset \Psi_{\mathrm{u}}$, is assumed to preserve the Poisson law as was endorsed via simulations in [4, Fig. 5]. Hence, it is reasonable to assume that the density of $\Psi$ is $\lambda_{\mathrm{b}}$ due to the OFDMA property.

As illustrated in Fig. 1, the distance from each active UE $x \in \Psi$ to its nearest BS $y \in \Psi_{\mathrm{b}}$ is denoted by $r_{x}$. Particularly, the distance from the typical BS $y_{0}$ to its serving UE $x_{0}$ is denoted by $r$. Besides, the distance from $y_{0}$ to the interfering UEs $x \in \Psi \backslash\left\{x_{0}\right\}$ is denoted by $d_{x}$.

\section{B. Channel Model and Power Control Scheme}

Multipath fading of the link between the typical BS $y_{0}$ and a UE $x$, is incorporated by a positive and i.i.d. Rayleigh fading $g_{x}$ with unit mean, i.e., $g_{x} \sim \exp (1)$. We consider a more realistic path loss model $\ell($.$) that can i) avoid the singularity { }^{1}$ at reduced transmit-receive distances [8] and ii) capture the subduction $^{2}$ effect of the path loss exponent [9], as

$$
\ell\left(r_{x}\right)=\left\{\begin{array}{cc}
\left(\delta+r_{x}^{\alpha_{0}}\right)^{-1} & , r_{x} \leq \mathrm{R}_{\mathrm{b}} \\
\mathrm{K} r_{x}^{-\alpha_{1}} & , r_{x}>\mathrm{R}_{\mathrm{b}}
\end{array}\right.
$$

where $\delta \in\{0,1\}, \mathbf{R}_{\mathrm{b}}$ is a baseline distance fitted from the propagation environment [9], $\mathrm{K}=\mathrm{R}_{\mathrm{b}}^{\alpha_{1}} /\left(\delta+\mathrm{R}_{\mathrm{b}}^{\alpha_{0}}\right)$ to ensure the continuity of $\ell($.$) in R_{b}$, and $\alpha_{0}, \alpha_{1}$ are respectively the near- and far-field path loss exponents, such as $0<\alpha_{0} \leq \alpha_{1}$.

(1) as defined above, is a unified framework that can capture several popular models of the path loss. Typically,

- $\ell($.$) is the standard unbounded path loss model (UPM)$ when $\delta=0$ and $\alpha_{0}=\alpha_{1}>2$ [4], [5].

- $\ell($.$) is the bounded path loss model (BPM) when \delta=1$ and $\alpha_{0}=\alpha_{1}>2$ [8].

\footnotetext{
${ }^{1}$ Holds when considering the standard path loss model of the form $r^{-\alpha}$.

${ }^{2}$ It is the variation of the path loss exponent in multi-breakpoint between the transmitter and the receiver.
}

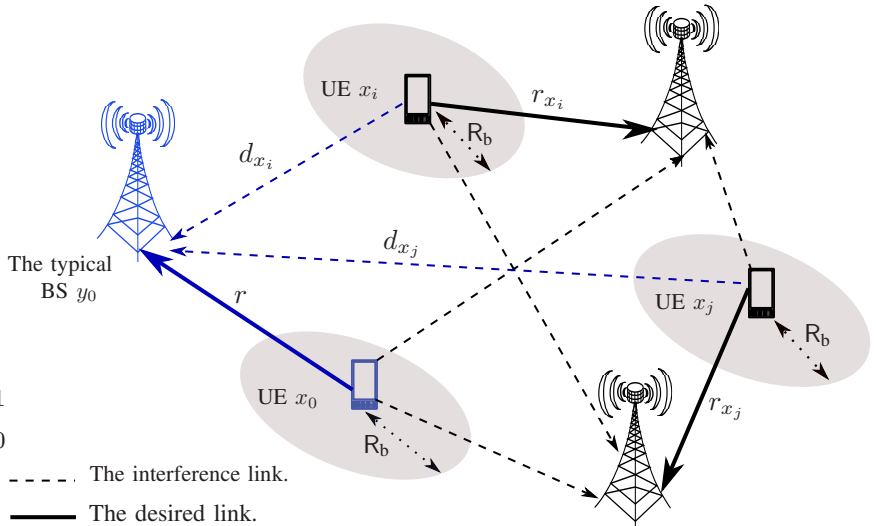

Fig. 1. The BS $y_{0}$ is served by $x_{0}$ and jammed by signals from the other active UEs. $R_{b}$ is a parameter of the path loss model.

- $\ell($.$) is the dual-slope path loss model (DSPM) when \delta=$ 0 and $\alpha_{0}<\alpha_{1}$ [9].

Since UEs are battery-powered, each UE needs to tune its transmit power and compensate for the path loss effect in accordance with the distance to its associated BS. Also, UE transmit power cannot be increased indefinitely, but needs to be bounded by a maximum value $P_{\max }$. We then introduce a specific power control scheme, namely the dual-slope bounded power control (DSBPC) function $\psi($.$) , as$

$$
\psi\left(r_{x}\right)=\left\{\begin{array}{ll}
\min \left\{\hat{P}_{\max } ;\left(\delta+r_{x}^{\alpha_{0}}\right)^{\epsilon}\right\}, & r_{x} \leq \mathrm{R}_{\mathrm{b}} \\
\min \left\{\hat{P}_{\max } ;\left(r_{x}^{\alpha_{1}} / \mathrm{K}\right)^{\epsilon}\right\}, & r_{x}>\mathrm{R}_{\mathrm{b}}
\end{array},\right.
$$

where $\epsilon \in\left[0, \epsilon_{\mathrm{d}}\right]$ is a parameter to tune the intensity of power control, such as $\epsilon_{\mathrm{d}} \geq 1$ is a design upper allowed value of $\epsilon$. $\hat{P}_{\max }$ is the UE maximum transmit power, normalized by $P_{\text {ref }}$ (UE reference transmit power when $\epsilon=0$ or $r_{x}=1-\delta$ ).

Since most UDNs are interference-limited, we focus our analysis on the uplink SIR at the typical BS $y_{0}$, expressed as

$$
\operatorname{SIR}\left(x_{0} ; y_{0}\right)=\frac{g_{x_{0}} \ell(r) \psi(r)}{I_{x_{0}}},
$$

where $I_{x_{0}}$ is the other-UE interference conditioned on a serving UE located at $x_{0}$, and expressed as

$$
I_{x_{0}}=\sum_{x \in \Psi \backslash\left\{x_{0}\right\}} g_{x} \ell\left(d_{x}\right) \psi\left(r_{x}\right) .
$$

\section{Distribution of Link Distances and the Process of the Interference Field}

Approximating the distribution of key distances $r$ and $r_{x}$, and characterizing the process of interfering UEs $\Psi \backslash\left\{x_{0}\right\}$, is generally challenging in uplink networks. This is in particular due to i) the coupling of active UEs location given the assumption of full-load uplink scenario combined with the OFDMA property, and ii) the coupling of active UEs location with that of BSs, due to the location dependent and power constrained DSBPC scheme. 
A tight approximation of active UEs point process is proposed in [14], but with limited change to system design insights. Hence, for tractability, we adopt similar generative assumptions as those considered in [4], [5], where the PDF of $r$ is expressed from the void probability as,

$$
f_{r}(\xi)=2 \pi \lambda_{\mathrm{b}} \exp \left(-\pi \lambda_{\mathrm{b}} \xi^{2}\right),
$$

and the distribution of $r_{x}$ conditioned on $d_{x}$, expressed under a truncated version of (5), as

$$
f_{r_{x}}\left(u \mid d_{x}\right)=\frac{f_{r}(u)}{1-\exp \left(-\pi \lambda_{\mathrm{b}} d_{x}^{2}\right)}, 0 \leq u \leq d_{x} .
$$

Furthermore, we model the location of interfering UEs by an inhomogeneous PPP outside an exclusion region of radius $r$. Its density is obtained as [4]

$$
\lambda_{I_{x_{0}}}\left(d_{x}\right)=\lambda_{\mathrm{b}}\left(1-\exp \left(-\pi \lambda_{\mathrm{b}} d_{x}^{2}\right)\right), \quad d_{x}>r .
$$

\section{Mobility Model}

In realistic scenarios where users QoE is a crucial metric, active UEs are constantly on a quest for more advantageous locations enabling optimal uplink SIR such as openings, windows, and elevated points in obstructed areas. Accordingly, active UEs are assumed to simultaneously engage in a random movement in $\mathbb{R}^{2}$ without prior knowledge of their nearest BS location, namely the blind cell search (BCS) movement. For tractability, we adopt the 3GPP simulation mobility model introduced in [13], wherein the UE moves in a straight line with velocity $v$ (distance per unit time), at angle $\theta$ w.r.t. the direction of connection. $\theta$ is randomly and uniformly distributed in $[0, \pi]$ due to symmetry.

\section{Uplink Coverage Probability Analysis}

In this section, we develop the baseline uplink framework under the DSBPC scheme (2). Typically, we consider two cases of analysis, i) the case of stationary active UEs and ii) the case of moving active UEs.

\section{A. Case of Stationary Active UEs}

When active UEs are randomly scattered but stationary, the uplink coverage probability is defined as the probability that the SIR at a typical BS located at the origin, exceeds a target T. Formally,

$$
\mathrm{P}_{\text {cov }}\left(\lambda_{\mathrm{b}}, \mathrm{T}\right)=\mathbb{P}\left(\mathrm{SIR}\left(x_{0} ; y_{0}\right) \geq \mathrm{T}\right),
$$

The following theorem derives the uplink coverage probability under the DSBPC scheme.

Theorem 1. The uplink coverage probability under the DS$B P C$ scheme is expressed as

$$
\begin{aligned}
\mathrm{P}_{c o v}\left(\lambda_{b}, \mathrm{~T}\right)=2 \pi \lambda_{b} & \int_{0}^{\mathrm{R}_{b}} r \exp \left(-\pi \lambda_{b} r^{2}\right) \Omega(r) \mathrm{d} r \\
& +2 \pi \lambda_{b} \int_{\mathrm{R}_{b}}^{\infty} r \exp \left(-\pi \lambda_{b} r^{2}\right) \Theta(r) \mathrm{d} r
\end{aligned}
$$

where the supplementary equations are listed in the top of the next page.
Proof. Given the formulation of SIR in (3), the definition of coverage probability in (8) is simplified as

$$
\mathrm{P}_{\text {cov }}\left(\lambda_{\mathrm{b}}, \mathrm{T}\right)=\mathbb{E}_{r}\left\{\mathcal{L}_{I_{x_{0}}}\left(\frac{\mathrm{T}}{\ell(r) \psi(r)} \mid r\right)\right\},
$$

where the inner conditional Laplace function is derived as

$$
\begin{aligned}
& \mathcal{L}_{I_{x_{0}}}\left(\frac{\mathrm{T}}{\ell(r) \psi(r)} \mid r\right)=\mathbb{E}\left\{\prod_{x \in \Psi \backslash\left\{x_{0}\right\}} \exp \left(-\frac{\mathrm{T} g_{x} \ell\left(d_{x}\right) \psi\left(r_{x}\right)}{\ell(r) \psi(r)}\right) \mid r\right\} \\
& \stackrel{(\mathrm{a})}{=} \mathbb{E}_{\Psi \backslash\left\{x_{0}\right\}}\left\{\prod_{x \in \Psi \backslash\left\{x_{0}\right\}} \mathbb{E}_{r_{x}}\left\{\frac{1}{1+\frac{\mathrm{T} \ell\left(d_{x}\right) \psi\left(r_{x}\right)}{\ell(r) \psi(r)}} \mid d_{x}, r\right\}\right\} \\
& \stackrel{(\mathrm{b})}{=} \exp \left(-2 \pi \lambda_{\mathrm{b}} \int_{r}^{\infty} \mathbb{E}_{r_{x}}\left\{\frac{1-\exp \left(-\pi \lambda_{\mathrm{b}} u^{2}\right)}{1+\frac{\ell(r) \psi(r)}{\mathrm{T} \ell(u) \psi\left(r_{x}\right)}} \mid r\right\} u \mathrm{~d} u\right), \text { (14) }
\end{aligned}
$$

where (a) holds since $\Psi \backslash\left\{x_{0}\right\}, r_{x}$, and $g_{x}$ are independent, in addition to $g_{x} \sim \exp (1)$, while (b) follows from the PGFL theorem [3, Prop. 1.2.2] and the expression of the interference process density in (7).

Due to paper brevity, the remainder of the proof is obtained by first substituting (1) and (2) in (14), and next using PDFs (5) and (6) to average over $r$ and $r_{x}$ conditioned on $d_{x}$.

Although the expression of the uplink coverage probability under the DSBPC scheme in (1) is in complicated form, it is general enough to accommodate several previous expressions in [4]-[6]. For brevity, developing special closed form expressions of (1) is deferred to the journal version.

\section{B. Case of Moving Active UEs}

To quantify the contribution of the BCS mobility model on the statistics of the uplink coverage probability at the level of the typical BS $y_{0}$, we introduce the induced ${ }^{3}$ uplink coverage probability, defined as the resultant uplink coverage probability following the BCS mobility model. Formally,

$$
\begin{aligned}
& \mathrm{P}_{\text {ind }}\left(\lambda_{\mathrm{b}}, \mathrm{T}, v\right)=\mathbb{E}_{r, \theta}\left(\mathbb{P}\left(\operatorname{SIR}\left(x ; y_{0}\right) \geq \mathrm{T}, \overline{\mathrm{h}} \mid r, \theta\right)\right) \\
& =\mathbb{E}_{r, \theta}\left\{\mathcal{L}_{I_{x}}\left(\frac{\mathrm{T}}{\ell(\xi) \psi(\xi)} \mid r, \theta\right) \mathrm{P}_{\overline{\mathrm{h}}}\left(\lambda_{\mathrm{b}}, v \mid r, \theta\right)\right\},
\end{aligned}
$$

where $x$ is the new location of the serving UE after the BCS such that $\xi=\left\|x-y_{0}\right\|=\sqrt{r^{2}+v^{2}+2 r v \cos (\theta)}, \overline{\mathrm{h}}$ denotes the event of no handover occurred where $\mathrm{P}_{\overline{\mathrm{h}}}\left(\lambda_{\mathrm{b}}, v \mid r, \theta\right)$ is the probability that the handover does not occur given $r$ and $\theta$.

In the following, we will first evaluate the uplink handoff rate before deriving the induced uplink coverage probability.

1) The handoff rate abstraction: OFDMA is very sensitive to uplink interference [15], where the SIR achieved by a given UE may be severely limited by signals from closer UEs to the tagged BS. In such context, the serving UE is more likely to trigger a handoff towards another BS. Accordingly, we consider an association-based handoff rate as in [12], wherein

\footnotetext{
${ }^{3} \mathrm{~A}$ dualism premise can be thought with the induced current in a coil following a random movement of the magnetic field inside it, i.e., the Faraday's law of electromagnetic induction.
} 


$$
\begin{aligned}
& \Omega(w)=\exp \left(-4 \pi^{2} \lambda_{\mathrm{b}}^{2}\left[\int_{w}^{\mathrm{R}_{\mathrm{b}}} h_{1}(u, w) u \mathrm{~d} u+\int_{\mathrm{R}_{\mathrm{b}}}^{\infty} h_{2}(u, w) u \mathrm{~d} u+\int_{\mathrm{R}_{\mathrm{b}}}^{\infty} h_{3}(u, w) u \mathrm{~d} u\right]\right), \\
& \Theta(w)=\exp \left(-4 \pi^{2} \lambda_{\mathrm{b}}^{2}\left[\int_{w}^{\infty} h_{4}(u, w) u \mathrm{~d} u+\int_{w}^{\infty} h_{5}(u, w) u \mathrm{~d} u\right]\right) \\
& h_{1}(u, w)=\int_{0}^{u} \frac{z \exp \left(-\pi \lambda_{\mathrm{b}} z^{2}\right)}{1+\frac{1}{\mathrm{~T}} \frac{\delta+u^{\alpha_{0}}}{\delta+w^{\alpha} 0} \frac{\min \left\{\hat{P}_{\max } ;\left(\delta+w^{\alpha_{0}}\right)^{\epsilon}\right\}}{\min \left\{\hat{P}_{\max } ;\left(\delta+z^{\alpha_{0}}\right)^{\epsilon}\right\}}} \mathrm{d} z, \quad h_{2}(u, w)=\int_{0}^{\mathrm{R}_{\mathrm{b}}} \frac{z \exp \left(-\pi \lambda_{\mathrm{b}} z^{2}\right)}{1+\frac{1}{\mathrm{TK}} \frac{u^{\alpha} 1}{\delta+w^{\alpha} 0} \frac{\min \left\{\hat{P}_{\max } ;\left(\delta+w^{\alpha_{0}}\right)^{\epsilon}\right\}}{\min \left\{\hat{P}_{\max } ;\left(\delta+z^{\alpha_{0}}\right)^{\epsilon}\right\}}} \mathrm{d} z,
\end{aligned}
$$

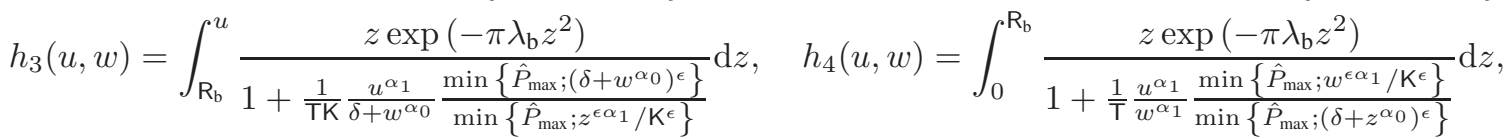

$$
\begin{aligned}
& h_{5}(u, w)=\int_{\mathrm{R}_{\mathrm{b}}}^{u} \frac{z \exp \left(-\pi \lambda_{\mathrm{b}} z^{2}\right)}{1+\frac{1}{\mathrm{~T}} \frac{u^{\alpha_{1}}}{w^{\alpha_{1}}} \frac{\min \left\{\hat{P}_{\max } ; w^{\epsilon \alpha_{1}} / \mathrm{K}^{\epsilon}\right\}}{\min \left\{\hat{P}_{\max } ; z^{\epsilon \alpha_{1}} / \mathrm{K}^{\epsilon}\right\}}} \mathrm{d} z .
\end{aligned}
$$

the serving UE triggers immediately a handoff event as soon as another active UE becomes more closer to the typical BS.

Proposition 1 in the top of the following page, derives the uplink handoff rate conceived as the probability of generating a handoff event at the typical BS.

Proof. Due to paper brevity, we will only give the outline of the proof. In fact, conditioned on $r$ and $\theta$, the probability that no handover occurs is expressed via the void probability, as

$$
\mathrm{P}_{\overline{\mathrm{h}}}\left(\lambda_{\mathrm{b}}, v \mid r, \theta\right)=\left\{\begin{array}{cl}
\mathrm{e}^{-\pi \lambda_{\mathrm{b}}\left(\xi^{2}-r^{2}\right)}, & 0 \leq \theta \leq \frac{\pi}{2} \& r \geq 0 \\
1, & \frac{\pi}{2} \leq \theta \leq \pi \& r \geq \frac{-v}{2 \cos (\theta)} \\
\mathrm{e}^{-\pi \lambda_{\mathrm{b}}\left(\xi^{2}-r^{2}\right)}, & \frac{\pi}{2} \leq \theta \leq \pi \& r \leq \frac{-v}{2 \cos (\theta)}
\end{array}\right.
$$

Next $\mathrm{P}_{\overline{\mathrm{h}}}\left(\lambda_{\mathrm{b}}, v\right)$ is derived by averaging over the distribution of $r$ in (5) and the distribution of $\theta$, assumed to be uniformly distributed in $[0, \pi]$ due to symmetry. We have then

$$
\begin{aligned}
& \mathrm{P}_{\overline{\mathrm{h}}}\left(\lambda_{\mathrm{b}}, v\right)=\mathbb{E}_{r, \theta}\left(\mathrm{P}_{\overline{\mathrm{h}}}\left(\lambda_{\mathrm{b}}, v \mid r, \theta\right)\right) \\
& =\frac{1}{\pi} \int_{0}^{\frac{\pi}{2}} \int_{0}^{\infty} 2 \pi \lambda_{\mathrm{b}} r \mathrm{e}^{-\pi \lambda_{\mathrm{b}} \xi^{2}} \mathrm{~d} r \mathrm{~d} \theta+\frac{1}{\pi} \int_{\frac{\pi}{2}}^{\pi} \int_{\frac{-v}{2 \cos (\theta)}}^{\infty} 2 \pi \lambda_{\mathrm{b}} r \mathrm{e}^{-\pi \lambda_{\mathrm{b}} r^{2}} \mathrm{~d} r \mathrm{~d} \theta \\
& +\frac{1}{\pi} \int_{\frac{\pi}{2}}^{\pi} \int_{0}^{\frac{-v}{2 \cos (\theta)}} 2 \pi \lambda_{\mathrm{b}} r \mathrm{e}^{-\pi \lambda_{\mathrm{b}} \xi^{2}} \mathrm{~d} r \mathrm{~d} \theta .
\end{aligned}
$$

The proof is finished by considering the definition of the error function $\operatorname{erf}($.$) and deriving the handoff probability as$ $\mathrm{P}_{\mathrm{h}}\left(\lambda_{\mathrm{b}}, v\right)=1-\mathrm{P}_{\overline{\mathrm{h}}}\left(\lambda_{\mathrm{b}}, v\right)$.

2) The induced coverage probability: Theorem 2 in the top of next page, derives the induced uplink coverage probability following the BCS movement.

Proof. Given the properties of the BCS mobility model as described in Section II-D, it is quite obvious to mention that the process of active UEs $\Psi$ remains a PPP. Also, given the expression of the induced uplink coverage probability in (16), we obtain

$$
\begin{aligned}
& \mathrm{P}_{\text {ind }}(.)=\frac{1}{\pi} \int_{0}^{\pi} \int_{0}^{\infty} 2 \pi \lambda_{\mathrm{b}} r \mathrm{e}^{-\pi \lambda_{\mathrm{b}} r^{2}} \mathrm{P}_{\overline{\mathrm{h}}}\left(\lambda_{\mathrm{b}}, v \mid r, \theta\right) \\
& \times \exp \left(-2 \pi \lambda_{\mathrm{b}} \int_{\xi}^{\infty} \mathbb{E}_{r_{x}}\left\{\frac{1-\exp \left(-\pi \lambda_{\mathrm{b}} u^{2}\right)}{1+\frac{\ell(\xi) \psi(\xi)}{\mathrm{T} \ell(u) \psi\left(r_{x}\right)}} \mid r, \theta\right\} u \mathrm{~d} u\right) \mathrm{d} r \mathrm{~d} \theta .
\end{aligned}
$$

Next, given the distance-dependence of the path loss model in (1) and the DSBPC in (2), splitting the expressions of $\ell(\xi)$ and $\psi(\xi)$ in (22) requires to previously identify the intervals of $r$ and $\theta$ that yield a greater or lower $\xi$ as compared to $\mathrm{R}_{\mathrm{b}}$, which comes to compare $\left(\frac{r}{v}+\cos (\theta)\right)^{2}-\left(\frac{\mathrm{R}_{b}^{2}}{v^{2}}-\sin ^{2}(\theta)\right)$ with 0 . Table I describes the effect of $r, \theta$, and $v$ intervals on the magnitude of $\xi$ w.r.t. $\mathrm{R}_{\mathrm{b}}$. We conclude the proof by combining Table. I and Eq. (22).

\begin{tabular}{|l|l|c|}
\cline { 2 - 3 } \multicolumn{1}{c|}{} & \multicolumn{1}{c|}{$v<\mathrm{R}_{\mathrm{b}}$} & \multicolumn{1}{c|}{$v \geq \mathrm{R}_{\mathrm{b}}$} \\
\hline$\xi \leq \mathrm{R}_{\mathrm{b}}$ & $0 \leq \theta \leq \frac{\pi}{2}, 0 \leq r \leq r_{0}$ & - \\
& $\frac{\pi}{2} \leq \theta \leq \pi, 0 \leq r \leq r_{1}$ & \\
& $\frac{\pi}{2} \leq \theta \leq \pi, \frac{-v}{2 \cos (\theta)} \leq r \leq r_{2}$ & \\
\hline$\xi \geq \mathrm{R}_{\mathrm{b}}$ & $0 \leq \theta \leq \frac{\pi}{2}, r \geq r_{0}$ & $0 \leq \theta \leq \frac{\pi}{2}, r \geq 0$ \\
& $\frac{\pi}{2} \leq \theta \leq \pi, r_{1} \leq r \leq \frac{-v}{2 \cos (\theta)}$ & $\frac{\pi}{2} \leq \theta \leq \pi, 0 \leq r \leq \frac{-v}{2 \cos (\theta)}$ \\
& $\frac{\pi}{2} \leq \theta \leq \pi, r \geq r_{2}$ & $\frac{\pi}{2} \leq \theta \leq \pi, r \geq \frac{-v}{2 \cos (\theta)}$ \\
\hline \multicolumn{2}{|c|}{ TABLE I }
\end{tabular}

COMPARING $\xi$ AND $\mathrm{R}_{\mathrm{B}}$ BASED ON THE INTERVALS OF $v, r$, AND $\theta$.

\section{NumERICAL RESUlts AND Discussions}

In this section, we present numerical results to assess our theoretical findings and quantify the interplay of system parameters. Numerical integration is evaluated using Matlab and Monte Carlo simulations are performed with $10^{5}$ iterations.

\section{A. Validation of the Analytical results}

Considering the three special cases of (1), namely the BPM, the UPM, and the DSPM, Fig. 2 describes the scaling of 
Proposition 1. The uplink handoff rate for a serving UE moving according to the BCS mobility model is expressed as

$$
\begin{aligned}
& \mathrm{P}_{h}\left(\lambda_{b}, v\right)=1-\exp \left(-\pi \lambda_{b} v^{2}\right)+v \sqrt{\lambda_{b}} \int_{\pi / 2}^{\pi} \cos (\theta) \mathrm{e}^{-\pi \lambda_{b} v^{2} \sin ^{2}(\theta)} \operatorname{erf}\left(v \sqrt{\pi \lambda_{b}} \frac{\cos (2 \theta)}{2 \cos (\theta)}\right) \mathrm{d} \theta \\
& +v \sqrt{\lambda_{b}} \int_{0}^{\pi / 2} \cos (\theta) \mathrm{e}^{-\pi \lambda_{b} v^{2} \sin ^{2}(\theta)} \mathrm{d} \theta-v \sqrt{\lambda_{b}} \int_{0}^{\pi} \cos (\theta) \mathrm{e}^{-\pi \lambda_{b} v^{2} \sin ^{2}(\theta)} \operatorname{erf}\left(v \sqrt{\pi \lambda_{b}} \cos (\theta)\right) \mathrm{d} \theta .
\end{aligned}
$$

Theorem 2. The induced uplink coverage probability at the typical BS following the BCS mobility model of active UEs, is expressed when $v<\mathrm{R}_{b}$, as

$$
\begin{aligned}
\mathrm{P}_{\text {ind }}\left(\lambda_{b}, \mathrm{~T}, v\right) & =2 \lambda_{b}\left[\int_{0}^{\frac{\pi}{2}} \int_{0}^{r_{0}} \mathrm{e}^{-\pi \lambda_{b} \xi^{2}} \Omega(\xi) r \mathrm{~d} r \mathrm{~d} \theta+\int_{\frac{\pi}{2}}^{\pi} \int_{0}^{r_{1}} \mathrm{e}^{-\pi \lambda_{b} \xi^{2}} \Omega(\xi) r \mathrm{~d} r \mathrm{~d} \theta+\int_{\frac{\pi}{2}}^{\pi} \int_{\frac{-v}{2 \cos (\theta)}}^{r_{2}} \mathrm{e}^{-\pi \lambda_{b} r^{2}} \Omega(\xi) r \mathrm{~d} r \mathrm{~d} \theta\right. \\
& \left.+\int_{0}^{\frac{\pi}{2}} \int_{r_{0}}^{\infty} \mathrm{e}^{-\pi \lambda_{b} \xi^{2}} \Theta(\xi) r \mathrm{~d} r \mathrm{~d} \theta+\int_{\frac{\pi}{2}}^{\pi} \int_{r_{1}}^{\frac{-v}{2 \cos (\theta)}} \mathrm{e}^{-\pi \lambda_{b} \xi^{2}} \Theta(\xi) r \mathrm{~d} r \mathrm{~d} \theta+\int_{\frac{\pi}{2}}^{\pi} \int_{r_{2}}^{\infty} \mathrm{e}^{-\pi \lambda_{b} r^{2}} \Theta(\xi) r \mathrm{~d} r \mathrm{~d} \theta\right],
\end{aligned}
$$

and when $v \geq \mathrm{R}_{b}$, as

$$
\mathrm{P}_{\text {ind }}\left(\lambda_{b}, \mathrm{~T}, v\right)=2 \lambda_{b}\left[\int_{0}^{\frac{\pi}{2}} \int_{0}^{\infty} \mathrm{e}^{-\pi \lambda_{b} \xi^{2}} \Theta(\xi) r \mathrm{~d} r \mathrm{~d} \theta+\int_{\frac{\pi}{2}}^{\pi} \int_{0}^{\frac{-v}{2 \cos (\theta)}} \mathrm{e}^{-\pi \lambda_{b} \xi^{2}} \Theta(\xi) r \mathrm{~d} r \mathrm{~d} \theta+\int_{\frac{\pi}{2}}^{\pi} \int_{\frac{-v}{2 \cos (\theta)}}^{\infty} \mathrm{e}^{-\pi \lambda_{b} r^{2}} \Theta(\xi) r \mathrm{~d} r \mathrm{~d} \theta\right],
$$

where $r_{0}=v \sqrt{\frac{\mathrm{R}_{b}^{2}}{v^{2}}-\sin ^{2}(\theta)}-v \cos (\theta), r_{1}=\min \left(r_{0}, \frac{-v}{2 \cos (\theta)}\right)$, and $r_{2}=\max \left(r_{0}, \frac{-v}{2 \cos (\theta)}\right)$.

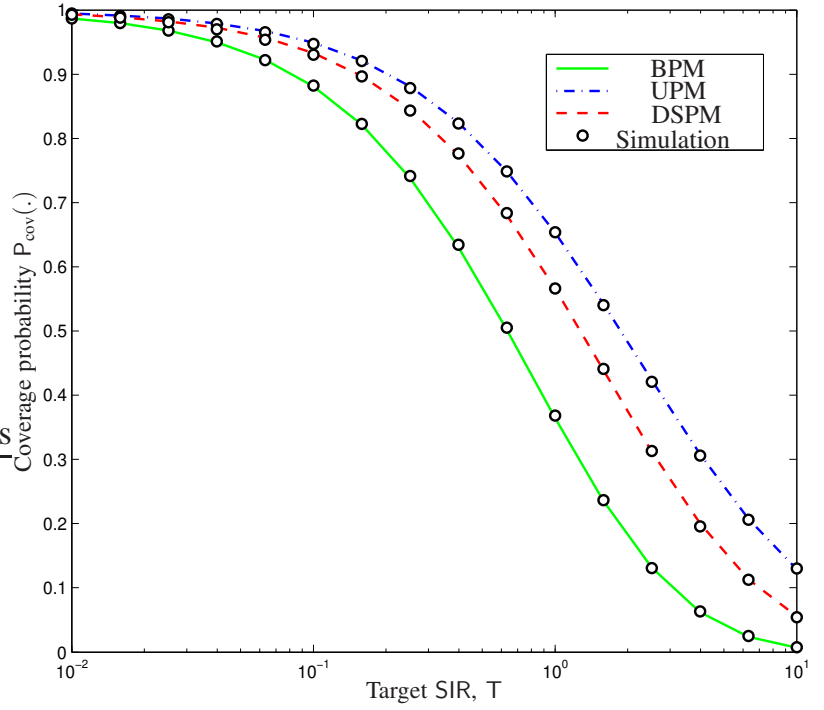

Fig. 2. The scaling of coverage probability with SIR threshold $T$ under the $\operatorname{BPM}\left(\delta=1, \alpha_{0}=\alpha_{1}=4\right)$, the UPM $\left(\delta=0, \alpha_{0}=\alpha_{1}=4\right)$, and the DSPM $\left(\delta=0, \mathrm{R}_{\mathrm{b}}=1, \alpha_{0}=2, \alpha_{1}=4\right)$. We use the following common parameters, $\hat{P}_{\max }=2, \lambda_{\mathrm{b}}=0.5$, and $\epsilon=0.7$.

the uplink coverage probability with the SIR threshold $\mathrm{T}$. The analytical curves (Theorem 1) match perfectly with the simulation results (markers), which validates the accuracy of our theoretical analysis. Typically, the UPM gives optimistic results of the uplink coverage probability as compared to BPM and DSPM, this is due in particular to the observation that the UPM amplifies the desired signal as $r \in[0,1]$.

\section{B. The DSBPC and the Uplink Performance of UDNs}

Based on cells density $\lambda_{\mathrm{b}}$, which implicitly reflects several loads of active uplink UEs, we can distinguish from Fig. 3, four operating regimes of OFDMA uplink networks.

1) The lightly loaded network regime: In such regime, e.g., $\lambda_{\mathrm{b}} \leq 10^{-2} \mathrm{BSs} / \mathrm{m}^{2}$ for $\hat{P}_{\max }=2$ in Figs. 3-(a), (b), and (c), the uplink coverage for fixed $\hat{P}_{\max }$, is i) similar under the three path loss models and ii) $\lambda_{\mathrm{b}}$-invariant regardless of $\epsilon$ (insensitive to power control). This is due to the observation that in such regime, UEs are more likely to be far from their nearest BS, and hence, the three path loss models are equivalent due to large transmitter-receiver distance. Also, UEs are more unlikely to benefit from the path loss compensation due to the $\hat{P}_{\max }$ constraint and hence the uplink interference power is counter-balanced by the serving signal power. We denote by $\lambda_{\mathrm{b}}^{(1)}$ the width of this regime. Interestingly, Figs. 3(d), (e), and (f) show that $\lambda_{\mathrm{b}}^{(1)}$ scales with $1 / \hat{P}_{\max }$.

2) The affluent regime: As $\lambda_{\mathrm{b}}$ becomes greater than $\lambda_{\mathrm{b}}^{(1)}$, we get into the affluent regime, wherein the uplink $\mathrm{P}_{\text {cov }}$ increases almost linearly with $\lambda_{\mathrm{b}}$ until a maximum value $P_{\text {cov }}^{\max }$ when $\lambda_{\mathrm{b}}$ achieves some $\lambda_{\mathrm{b}}^{(2)}$. This $\mathrm{P}_{\text {cov }}$ trend is particularly due to the increasing amount of active UEs that succeed to invert the path loss, while farther UEs (interferers) are still drastically constrained by $\hat{P}_{\max }$. Interestingly, we can sense the effect of $\epsilon$ in this regime and the gap between UPM, BPM, and DSPM begins to emerge. Typically, $P_{\text {cov }}$ remains almost unchanged from the previous regime if $\epsilon=0$ and an increasing $\epsilon$ improves the rate of $\mathrm{P}_{\mathrm{cov}}$ growth, until an optimum exponent $\epsilon_{\mathrm{opt}}<1$, after which the rate of $P_{\text {cov }}$ growth shrinks down. Numerically, we obtain from Figs. 3-(a), (b), and (c), that $\epsilon_{\text {opt }} \simeq 0.6$ under the UPM and DSPM, while $\epsilon_{\mathrm{opt}} \simeq 0.2$ under the BPM.

3) The decay regime: when $\lambda_{\mathrm{b}}>\lambda_{\mathrm{b}}^{(2)}$, the average transmitter-receiver distance decreases, and hence the amount of farther (interfereing) UEs that succeed to invert the path loss increases, while close UEs are increasingly reducing their 


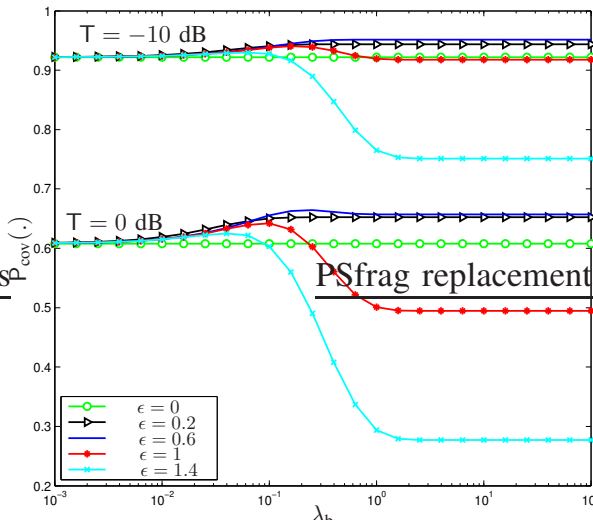

$\lambda_{\mathrm{b}}$
(a)

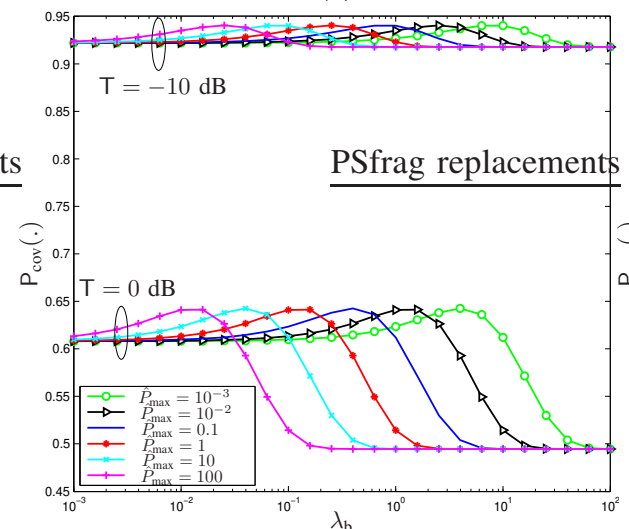

(d)

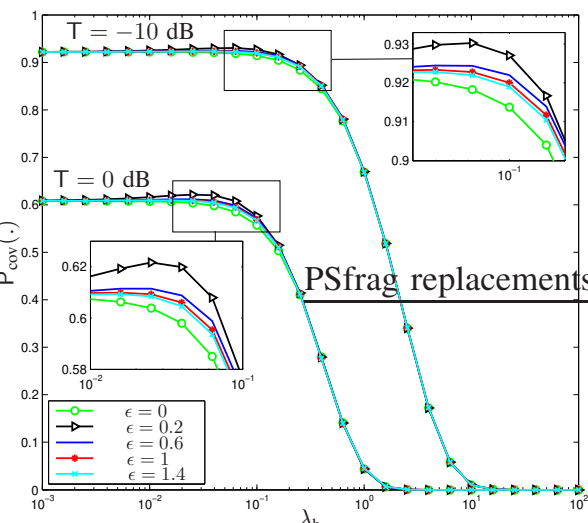

(b)

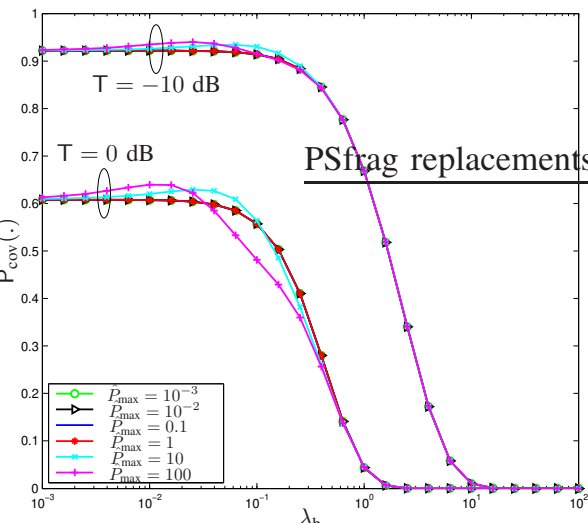

(e)

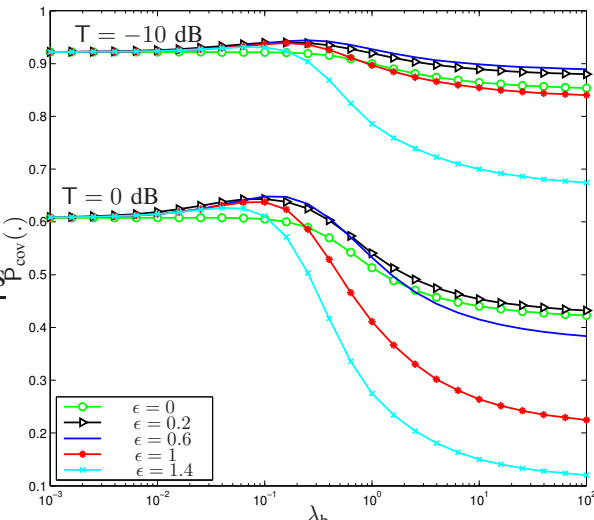

$\lambda_{\mathrm{b}}$
(c)

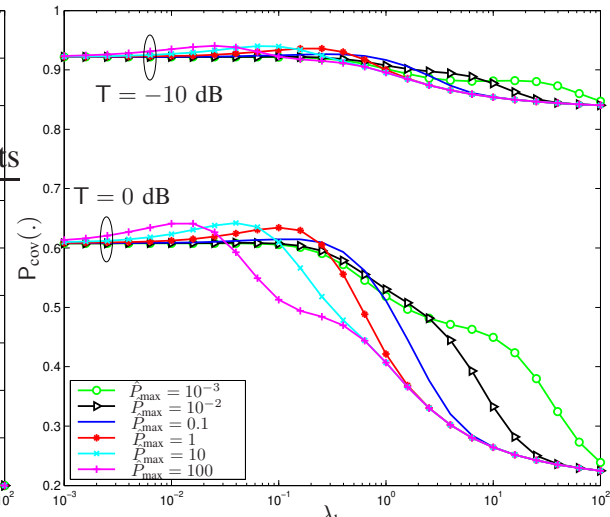

$\lambda_{b}$
(f)

Fig. 3. The scaling of the uplink coverage probability $\mathrm{P}_{\operatorname{cov}}($.$) as a function of BS density \lambda_{\mathrm{b}}$ and i) the power control exponent $\epsilon$ for $\hat{P}_{\max }=2$ in (a), (b) and (c). ii) the UE normalized maximum transmit power $\hat{P}_{\max }$ for $\epsilon=1$ in (d), (e) and (f). Parameters for the UPM case in (a) and (d), are $\alpha_{0}=\alpha_{1}=4$ and $\delta=0$, for the BPM case in (b) and (e), are $\alpha_{0}=\alpha_{1}=4$ and $\delta=1$, and for the DSPM case in (c) and (f), are $\alpha_{0}=3, \alpha_{1}=4, \mathrm{R}_{\mathrm{b}}=1$ and $\delta=0$.

transmit powers, particularly when $\epsilon \geq \epsilon_{\mathrm{opt}}$, which results on the decay of the uplink coverage probability. However, when $\epsilon<\epsilon_{\mathrm{opt}}$, the trend of uplink coverage probability is dependent on the considered path loss model. In fact, under the UPM, an increase in the interference power will be almost counter-balanced by an equivalent increase in the desired signal power due to singularity of the model, and hence the SIR remains almost invariant from the previous regime (Fig. 3(a)). Conversly, the uplink coverage probability will decay under the BPM and the DSPM where the interference will be more powerful than the desired signal power given the nonsingularity of the BPM, and the lower path loss exponent of the near-field region under the DSPM [9].

4) The UDNs regime: when $\lambda_{\mathrm{b}}$ is getting sufficiently large, e.g., $\lambda_{\mathrm{b}} \geq 1 \mathrm{BS} / \mathrm{m}^{2}$ for $\mathrm{T}=0 \mathrm{~dB}$ in Fig. 3, i.e., the context of UDNs, or heavily loaded OFDMA uplink networks. The gap between UPM, BPM and DSPM becomes evident. Typically, the SIR becomes $\lambda_{\mathrm{b}}$-invariant under the UPM, due to the equilibrium between the interference and the desired signal powers. Conversely, the uplink $\mathrm{P}_{\text {cov }}$ under the BPM goes towards the near-universal outage as $\lambda_{\mathrm{b}} \longrightarrow \infty$, due to non-singularity at the transmitter [8]. Also, the uplink $\mathrm{P}_{\text {cov }}$ under the DSPM, goes towards 0 as $\lambda_{\mathrm{b}} \longrightarrow \infty$ if $\alpha_{0} \leq 2$ (Fig. 3-(c)). Similar proof to [9, Prop. 1] is omitted here.

\section{The Impact of Mobility on the Uplink Performance}

Fig. 4 illustrates the scaling of the uplink handoff rate as a function of UE velocity $v$ and network density $\lambda_{\mathrm{b}}$. The analytical expression in Prop. 1 exactly matchs the simulation results, which validate the accuracy of the theoretical analysis. Also, the curves in Fig. 4 show that the uplink handoff rate increases naturally with UE velocity $v$ and cells density $\lambda_{\mathrm{b}}$.

In Fig. 5, we assess the induced uplink coverage probability of Theorem. 2 conditioned on $\theta$. Based on the parameter pair $(v, \theta)$, We can identify the following key cases.

1) A fleeing repulsive motion: it occurs when the serving UE moves away from $y_{0}$ at the earliest stage of its random movement, i.e., $0 \leq \theta \leq \pi / 2$. That is, the serving UE movement is more likely to reduce the uplink $P_{\text {cov }}$ under the UPM and BPM since it widens the desired link distance. Conversely, the DSPM enables to improve the induced coverage probability for low velocity of the serving UE, i.e., $0<v<\mathrm{R}_{\mathrm{b}}$, due particularly to the observation that such random movement will reduce the desired signal power for low $v$ and also the average number of interfering active UEs inside the near-field disk. However, when $v$ is high enough, i.e., $v>\mathrm{R}_{\mathrm{b}}$, the uplink $\mathrm{P}_{\text {cov }}$ under DSPM converges to that under the UPM with $\alpha_{1}$.

2) A fleeing clustering motion: it occurs when the serving UE moves towards $y_{0}$ and next gets away from it given the 


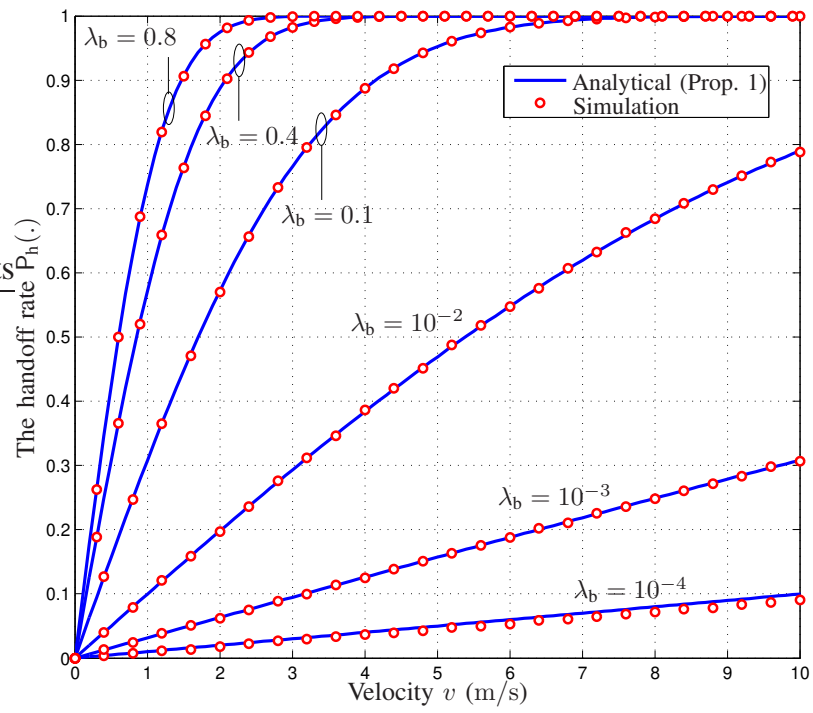

Fig. 4. The handoff rate as a function of the serving UE velocity $v$ and the BS density $\lambda_{\mathrm{b}}$.

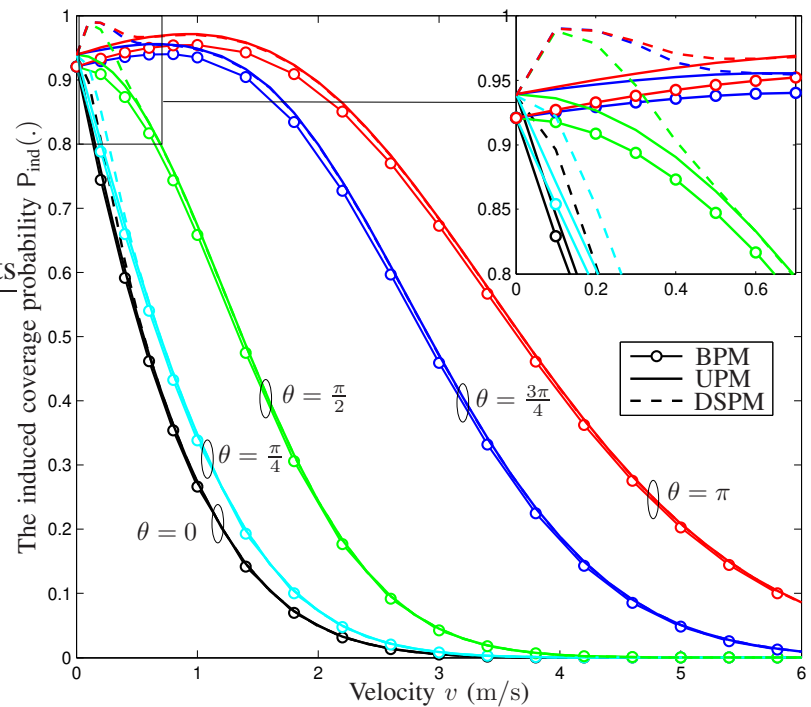

Fig. 5. The uplink induced coverage probability following the serving UE movement with velocity $v$, in the direction $\theta$ w.r.t. the link to the tagged BS We use the following parameters: $\lambda_{\mathrm{b}}=10^{-3}, \mathrm{~T}=-10 \mathrm{~dB}, \hat{P}_{\max }=2$, the $\operatorname{BPM}\left(\delta=1, \alpha_{0}=\alpha_{1}=4\right)$, the UPM $\left(\delta=0, \alpha_{0}=\alpha_{1}=4\right)$, and the $\operatorname{DSPM}\left(\delta=0, \mathrm{R}_{\mathrm{b}}=1, \alpha_{0}=3, \alpha_{1}=4\right)$.

amplitude of $v$, i.e., $\pi / 2<\theta \leq \pi$. In such a context, the uplink coverage probability under the UPM and the BPM, increases steadily with the serving UE mobility, until achieving the nearest allowed point to the typical BS. Next, the uplink $P_{\text {cov }}$ shrinks down until a handover occurs. A different performance trend is reported under the DSPM, where the uplink coverage probability significantly increases for low user velocity, due to the interplay between of the desired signal and the interference powers with the near-field disk, next the $P_{\text {cov }}$ curve converges to that under the UPM for sufficiently higher velocities.

\section{CONClusion}

Considering a novel power control scheme built upon realistic path loss models and UEs constrained transmit power, we first develop a unified mathematical framework of the uplink coverage probability assuming a randomly but stationary active UEs. Next, and in order to improve their connectivity conditions, active UEs are assumed to engage in a linear random movement according to the 3GPP simulation mobility model. In such a context, we develop a tractable expression of the uplink handoff rate, which is necessary to derive the induced uplink coverage probability following mobility model. Analytical results are validated via simulations where we have identified four operating regimes of OFDMA uplink networks depending on system design parameters and the path loss model considered.

Typically, it has been shown that for sufficiently dense networks, the impact of power control on the uplink coverage is limited under the BPM, particularly for low SIR target, where farther UEs are more likely to jam the desired signal regardless of the path loss compensation. Conversely, the impact of power control is meaningful under the UPM and the DSPM due to singularity at very low distances.

\section{REFERENCES}

[1] Ericsson Mobility Report on the Pulse of the Networked Society, Nov. 2016, Stockholm, Sweden. [Online]. Available: https://www.ericsson.com/assets/local/mobility-report/documents/2016/ ericsson-mobility-report-november-2016.pdf

[2] M. Kamel, W. Hamouda, and A. Youssef, "Ultra-dense networks: A survey," IEEE Commun. Surveys Tuts., vol. 18, no. 4, pp. 2522-2545, Fourthquarter 2016.

[3] F. Baccelli and B. Blaszczyszyn, Stochastic Geometry and Wireless Networks in Foundations and Trends in Networking, vol. 1, Now Publishers, 2009.

[4] J. G. Andrews, A. K. Gupta, and H. S. Dhillon, "A primer on cellular network analysis using stochastic geometry," arXiv preprint, Oct. 2016, [Online]. Available: https://arxiv.org/abs/1604.03183.

[5] T. D. Novlan, H. S. Dhillon, and J. G. Andrews, "Analytical modeling of uplink cellular networks," IEEE Trans. Wireless Commun., vol. 12 , no. 6, pp. 2669-2679, Jun. 2013.

[6] M. Di Renzo and P. Guan, "Stochastic geometry modeling and systemlevel analysis of uplink heterogeneous cellular networks with multiantenna base stations," IEEE Trans. Commun., vol. 64, no. 6, pp. 24532476, Jun. 2016.

[7] H. ElSawy and E. Hossain, "On stochastic geometry modeling of cellular uplink transmission with truncated channel inversion power control," IEEE Trans. Wireless Commun., vol. 13, no. 8, pp. 4454-4469, Aug. 2014.

[8] J. Liu, M. Sheng, L. Liu, and J. Li, "Effect of densification on cellular network performance with bounded pathloss model," IEEE Commun. Lett., vol. 21, no. 2, pp. 346-349, Feb. 2017.

[9] X. Zhang and J. G. Andrews, "Downlink cellular network analysis with multi-slope path loss models", IEEE Trans. Commun., vol. 63, no. 5, pp. 1881-1894, May 2015.

[10] T. Ding, M. Ding, G. Mao, Z. Lin, D. López-Pérez, and A. Y. Zomaya, "Uplink performance analysis of dense cellular networks with LoS and NLoS transmissions," IEEE Trans. Wireless Commun., vol. 16, no. 4, pp. 2601-2613, Apr. 2017.

[11] R. Arshad, H. Elsawy, S. Sorour, M. Alouini, and T. Y. Al-Naffouri, "Mobility-aware user association in uplink cellular networks," IEEE Commun. Lett., vol. 21, no. 11, pp. 2452-2455, Nov. 2017.

[12] S. Sadr and R. S. Adve, "Handoff rate and coverage analysis in multitier heterogeneous networks," IEEE Trans. Wireless Commun., vol. 14, no. 5, pp. 2626-2638, May 2015.

[13] 3GPP, "Enhanced LTE support for aerial vehicles," 3rd Generation Partnership Project (3GPP), Tech. Rep. 36.777, 01 2018, version 1.1.0.

[14] Y. Wang, M. Haenggi, and Z. Tan, "The meta distribution of the SIR for cellular networks with power control," IEEE Trans. Commun., vol. 66, no. 4, pp. 1745-1757, Apr. 2018.

[15] Y. Sun, R. P. Jover, and X. Wang, "Uplink interference mitigation for OFDMA femtocell networks," IEEE Trans. Wireless Commun., vol. 11, no. 2, pp. 614-625, Feb. 2012. 